A

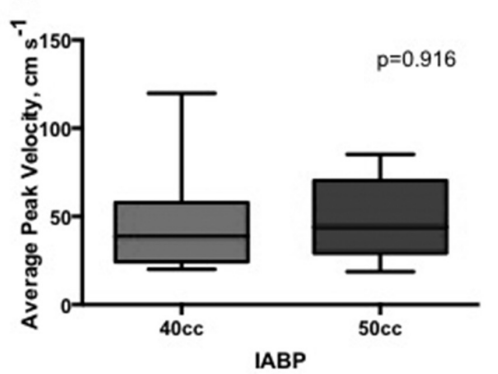

B

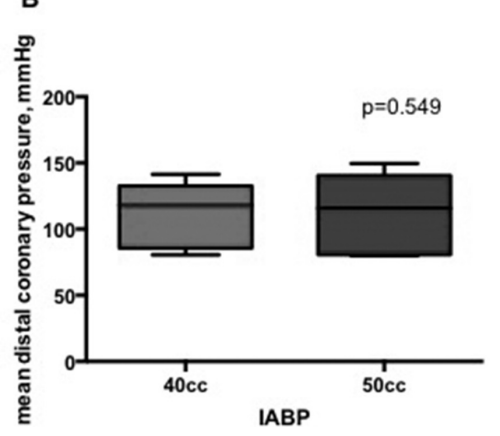

Abstract 21 Figure 1 Box plots demonstrating effects of 40cc and $50 \mathrm{cc}$ balloons on average peak velocity $(A)$ and mean distal coronary pressure (B).

\section{FACTORS ASSOCIATED WITH POOR OUTCOME IN PATIENTS TAKEN TO THE CATHETER LAB AFTER OUT OF HOSPITAL CARDIAC ARREST}

${ }^{1}$ Christian Camm*, ${ }^{2}$ Roshan Xavier, ${ }^{2}$ Rajesh Kharbanda, ${ }^{2}$ Sam Dawkins. 'University of Oxford; ${ }^{2}$ Oxford University Hospitals NHS Foundation Trust

\subsection{6/heartjnl-2017-311726.22}

Introduction Out of hospital cardiac arrest (OHCA) represents a common presentation to both the emergency department and the catheter lab. Understanding of the factors associated with poor outcome in this patient group is limited; thus management decisions are challenging. The aim of this analysis was to retrospectively review clinical records for OHCA patients undergoing catheter lab procedures to determine factors associated with poor outcome.

Methods Data on patients undergoing coronary angiography and percutaneous coronary intervention (PCI) between January 2009 and May 2015 at a tertiary cardiac centre were retrospectively reviewed. A keyword search was performed on all records to identify relevant procedures and these results were manually reviewed by two authors to confirm they were OHCA cases. Cases were excluded if they initially presented to a different hospital and were later transferred for investigation. Procedure details, discharge summaries, blood results and mortality data were reviewed.
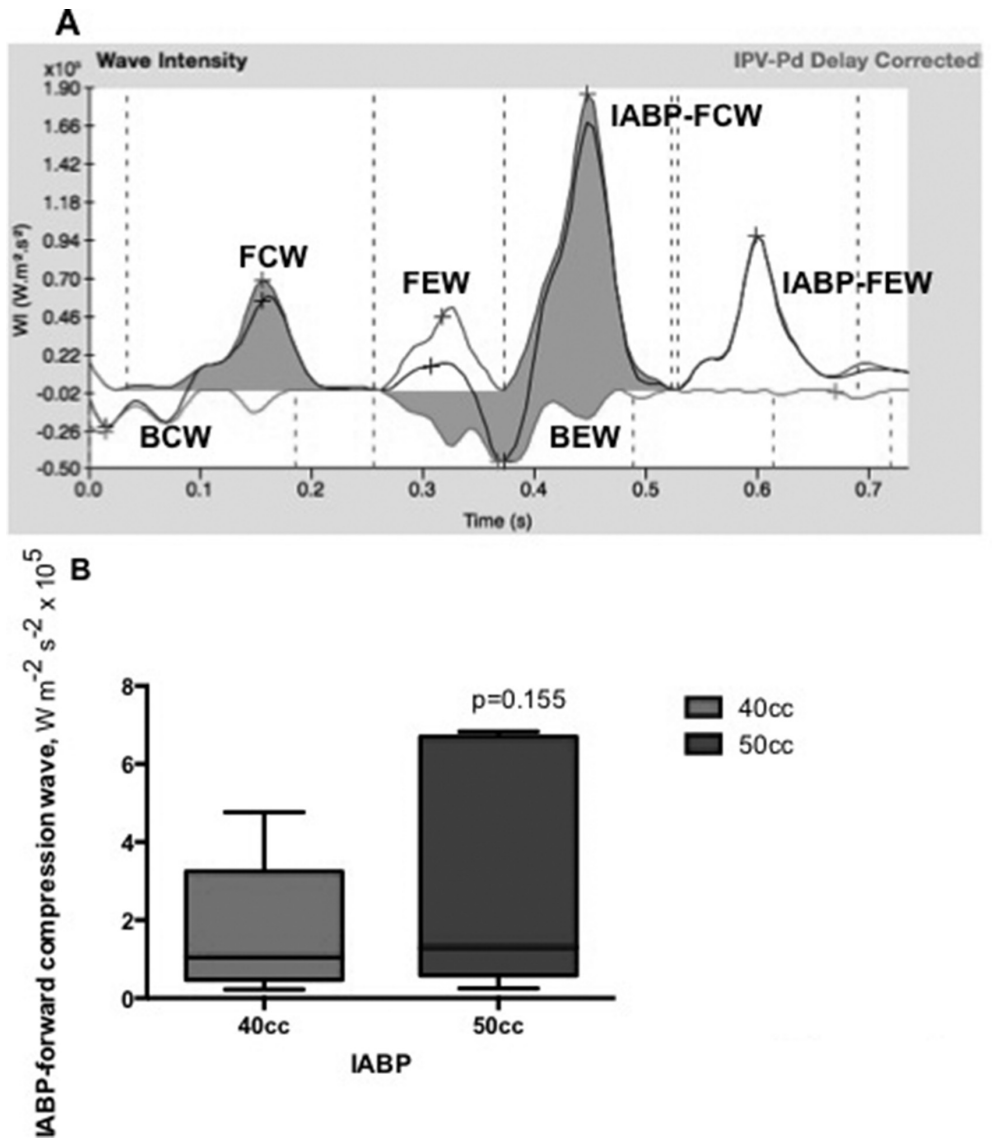

Abstract 21 Figure 2 (A) Wave Intensity Profile during intra-aortic balloon pump assisted conditions, highlighting the temporally related wave energies with device inflation (IABP-FCW) and deflation (IABP-FEW). FCW=forward compression wave; $B C W=$ backward compression wave; $\mathrm{FEW}=$ forward expansion wave, $\mathrm{BEW}=$ backward expansion wave. (B) Box plot demonstrating effects of $40 \mathrm{cc}$ and $50 \mathrm{cc}$ balloons on IABP-FCW during basal conditions. 
Abstract 22 Table 1 Demographic details of patients attending the catheter lab following OHCA. Values shown as mean \pm standard deviation or $\mathrm{n}(\%)$.

\begin{tabular}{ll}
\hline Item & Number of Patients \\
\hline Total OHCA Patients & 242 \\
Age (Years) & $62.2 \pm 13.0$ \\
Male & $190(78.5)$ \\
30 Day Mortality & $42(17.4)$ \\
1 Year Mortality & $47(19.4)$ \\
\hline
\end{tabular}

Abstract 22 Table 2 Selected prognostic factors associated with 30-day mortality in patients attending the catheter lab following OHCA. Values shown as n (\%). For the first row, percentage is of row total, in other rows percentages is that of column total.

\begin{tabular}{lllll}
\hline Item & Total & Dead & Alive & P-value \\
\hline All Patients & $242(100.0)$ & $42(17.4)$ & $200(82.6)$ & \\
Cardiogenic Shock & $57(23.6)$ & $22(52.4)$ & $35(17.5)$ & $<0.001$ \\
Any PEA ( $\mathrm{n}=228)$ & $25(11.0)$ & $10(25.6)$ & $15(7.9)$ & 0.001 \\
Intubated prior to catheter lab & $121(50.0)$ & $35(83.3)$ & $86(43.0)$ & $<0.001$ \\
ST-Elevation & $119(49.2)$ & $23(54.8)$ & $96(48.0)$ & 0.426 \\
Underwent PCI & $162(66.9)$ & $28(66.7)$ & $134(67.0)$ & 0.967 \\
\hline
\end{tabular}

Results 27578 angiogram or PCI procedures were carried out between January 2009 and May 2015; 242 (0.9\%) of these were patients presenting with OHCA. Forty-two patients $(17.3 \%)$ died within 30 days of presentation. Demographic details of this population are shown in Table 1. Univariate analysis revealed that blood gas $\mathrm{pH}$ and lactate were strongly correlated with 30 day mortality $(p<0.001$ and $p=0.002$ respectively). Other factors associated with 30 day mortality included presentation with cardiogenic shock, intubation prehospital or in the emergency department (but not in the

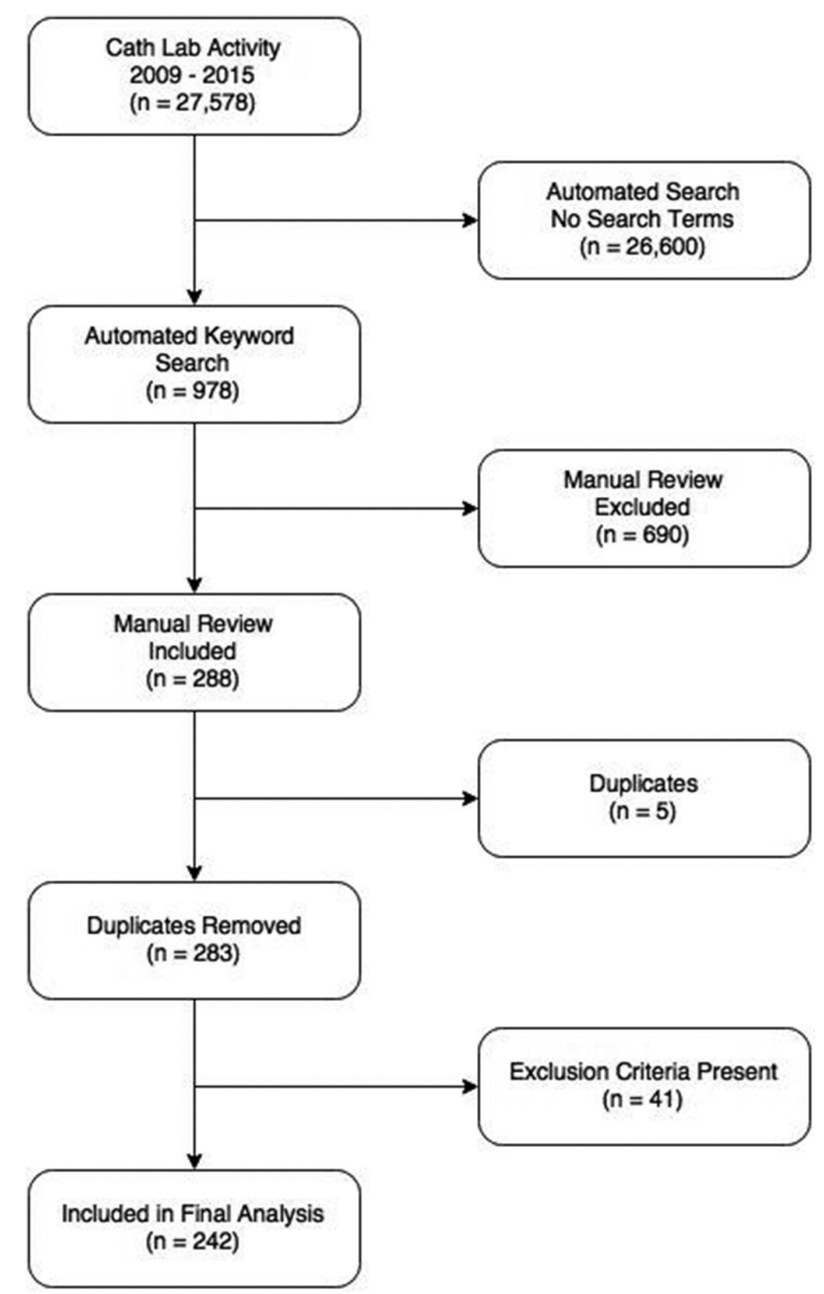

Abstract 22 Figure 1 Flow-chart detailing patient identification for this study.

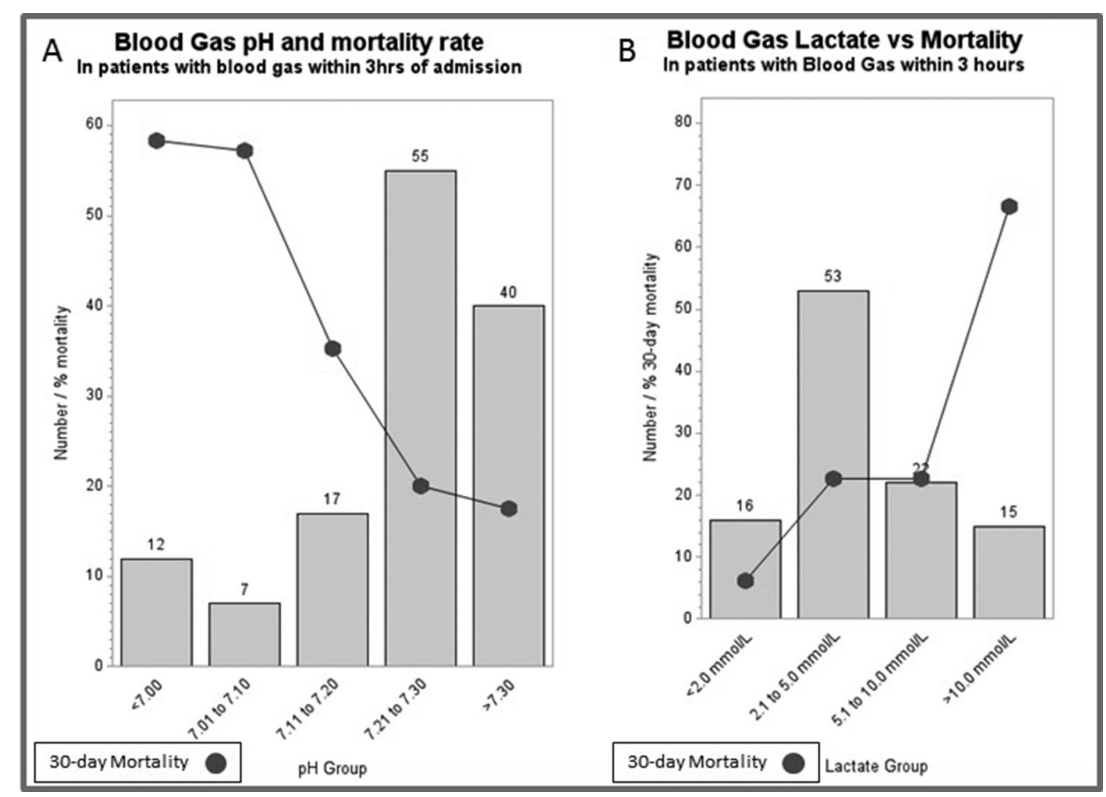

Abstract 22 Figure 2 Bar charts showing blood gas pH and lactate against 30-day mortality 
catheter lab) and the development of pulseless electrical activity (PEA) at any time (table 2$)$. Culprit vessel $(p=0.810)$, STelevation at presentation, and age were not significantly associated with 30 day mortality $(p=0.426$ and $p=0.085$ respectively). Furthermore, there was no difference in mortality between those who underwent PCI and those who received angiography alone.

Conclusion OHCA constituted $0.9 \%$ of activity during the period of review. There were significant correlations between 30 day mortality and several biochemical and clinical markers available at presentation. The use of these markers may be of use in triaging patients who are likely to benefit from interventional procedures.

\section{THE INFLUENCE OF COLLATERAL REGRESSION POST CORONARY CHRONIC TOTAL OCCLUSION (CTO) PERCUTANEOUS CORONARY INTERVENTION (PCI) ON DONOR VESSEL CORONARY PRESSURE-DERIVED MEASUREMENTS}

${ }^{1}$ Shah Mohdnazri* ${ }^{1}$ 'Firas Al-Janabi, ${ }^{1}$ Grigoris Karamasis, ${ }^{2}$ James Hampton-Till, ${ }^{3}$ Rasha AlLamee, 'Jason Dungu, ' ${ }^{1}$ Swamy Gedela, ${ }^{1}$ Kare Tang, ${ }^{1}$ Paul Kelly, ${ }^{3}$ Justin Davies, ${ }^{1}$ John Davies, ${ }^{1}$ Thomas Keeble. ${ }^{1}$ Essex Cardiothoracic Centre; ${ }^{2}$ Anglia Ruskin University; ${ }^{3}$ Imperial College London

\subsection{6/heartjnl-2017-311726.23}

Background There is strong evidence of FFR guided treatment in multi-vessel disease. The presence of a concomitant CTO may influence the FFR measurement in donor vessel as suggested in previous studies and reports. This has an important implication on clinical decision making for complete revascularisation in patients with chronic total occlusion. We sought to investigate the influence of collateral regression after successful CTO recanalisation on donor vessel pressure-derived indices.

Methods The study participants were patients with angina who had RCA CTO. 28 out of 34 consecutive patients underwent successful PCI to RCA CTOs during the study period and completed the follow study (at 3 months post CTO PCI) were included in this analysis. Coronary pressure-derived indices (resting PD/PA, iFR and FFR) were measured pre and post successful RCA CTO PCI in donor vessels and at follow up procedures.

Results The mean age was 62.38 years. The mean estimated CTO duration was 238.72 weeks and CTO length was $32.44 \mathrm{~mm}$. 25 patients had ischaemia and or viability in the RCA territory assessed with cardiac MRI. LAD was the major donor vessel in 24 patients and LCX was the minor donor vessel in 4 patients. Percent stenosis on QCA in the major and minor donor vessel were $40.6 \%$ and $35.1 \%$ respectively. The mean resting $\mathrm{Pd} / \mathrm{Pa}$, iFR and FFR pre and post RCA CTO PCI and at follow-up procedures in major donor vessel were $(0.893,0.862,0.764),(0.907,0.886,0.753)$ and $(0.918$, $0.901,0.787)$ respectively. The mean resting $\mathrm{Pd} / \mathrm{Pa}, \mathrm{iFR}$ and FFR pre and post RCA CTO PCI and at follow-up procedures in minor donor vessel were $(0.979,0.966,0.890)$, $(0.983,0.979,0.880)$ and $(0.981,0.974,0.898)$ respectively. The changes in coronary pressure-derived indices pre and post RCA CTO PCI and at follow up procedures are summarised in table 1. In major donor vessel, there was significant changes in the difference between follow up and pre-CTO PCI values for $\mathrm{Pd} / \mathrm{Pa}$, iFR and FFR values ( $\mathrm{p}$ values 0.006 , 0.003 and 0.047 respectively). There was also significant

Abstract 23 Table 1 Coronary pressure-derived indices pre and post RCA CTO PCI and at follow up procedures (FU:Follow-up, PCl: Percutaneous Coronary Intervention, FFR: Fractional Flow Reserve, СTO: Chronic Total Occlusion)

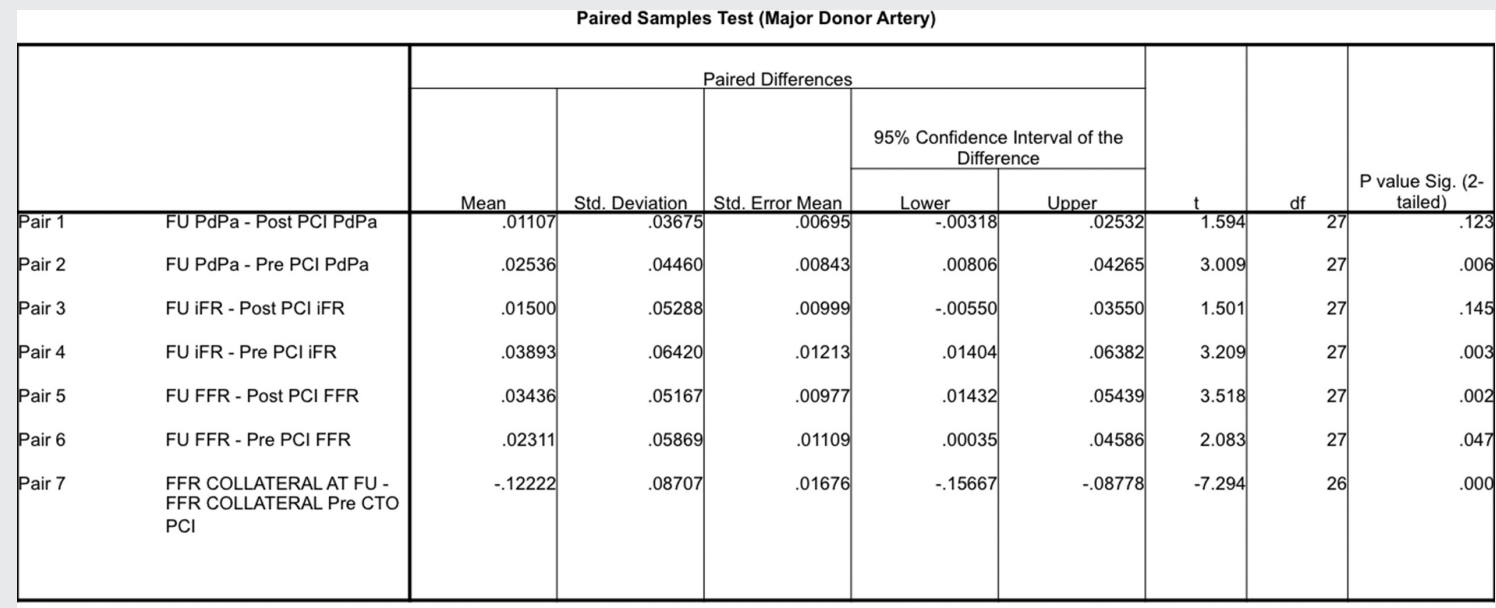

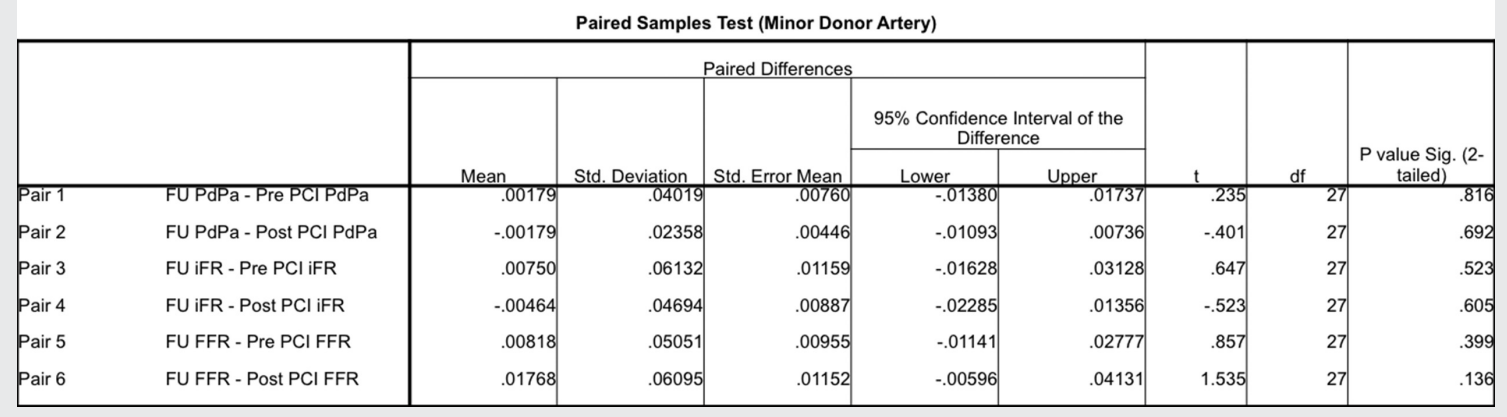

